Check for updates

Cite this: J. Mater. Chem. A, 2021, 9, 22380

Received 19th June 2021

Accepted 13th September 2021

DOI: 10.1039/d1ta05194f

rsc.li/materials-a

\title{
Insights into light and mass transport in nanoparticle-based aerogels: the advantages of monolithic 3D photocatalysts $\uparrow$
}

\author{
Ana Laura Luna, (D) *a Sotirios Papadopoulos, (D) b Till Kyburz, $^{a}$ Elena Tervoort, ${ }^{a}$ \\ Lukas Novotny ${ }^{b}$ and Markus Niederberger iD a
}

\begin{abstract}
The interest of nanoparticle-based aerogels-3-dimensional (3D) nanoparticle assemblies of macroscopic sizes -in photocatalysis is mainly their versatility for tailoring properties. However, little is known about the true viability of the aerogels as photocatalysts in part because of their 3D structure that is unusual for this application. Here, we investigate the mass and light transport effectiveness in the whole aerogel network and evaluate their photoactivity. We used anatase-based aerogels as model samples and designed a continuous-flow reactor to perform such analyses. It was found that the photons with suitable energy to initiate the reaction can propagate through the entire monolith because of their diffusive transport, making the 3D network fully active. The mass transport in the aerogels was also effective but is influenced by the reactor design. The aerogels showed promising activity, e.g., $\mathrm{Pd}$-anatase aerogels with a volume of only $0.5 \pm 0.04 \mathrm{~cm}^{3}$ generated $92 \mu \mathrm{mol} \mathrm{h} \mathrm{h}^{-1}$ of $\mathrm{H}_{2}$ with an apparent quantum efficiency of $13 \%$ at $375 \mathrm{~nm}$.
\end{abstract}

\section{Introduction}

Solar energy conversion plays an essential role in addressing the global warming challenge and energy demands of our times. Among the innovative ways to transform sunlight, photocatalysis stands out as a direct-conversion and potentially costeffective approach through which sunlight can be converted into solar fuels such as hydrogen (via water splitting or photoreforming) and hydrocarbons (via $\mathrm{CO}_{2}$ reduction). ${ }^{1-3}$ However, for the practical application of photocatalysis in solar fuel generation, highly efficient catalysts need to be developed. Due to the multiple tasks (light absorption, charge carrier separation, and surface redox reactions) that a photocatalyst has to perform simultaneously, it is difficult that one-component photocatalysts can achieve high efficiencies. ${ }^{\mathbf{4}-\mathbf{6}}$ Nevertheless, the limitation of a single semiconductor can be compensated by developing multicomponent materials, which have demonstrated to exhibit a higher photoactivity. In this regard, nanoparticle-based aerogels have triggered research attention as an innovative and powerful platform to design hybrid and highly active photocatalysts rationally. ${ }^{7-9}$

${ }^{a}$ Laboratory for Multifunctional Materials, Department of Materials, ETH Zürich, Vladimir-Prelog-Weg 5, 8093 Zürich, Switzerland. E-mail: ana.laura.luna.barron@ alumni.ethz.ch

${ }^{b}$ Photonics Laboratory, ETH Zürich, CH-8093 Zürich, Switzerland

$\dagger$ Electronic supplementary information (ESI) available. See DOI: $10.1039 / \mathrm{d} 1 \mathrm{ta} 05194 \mathrm{f}$
Nanoparticles-based aerogels are advanced materials formed by nanoparticles assembled into a 3D porous network. The network allows the interaction between the nanoparticles without harming their size-dependent properties. If the network is a single body, a so-called monolith, in the size range of centimeters, then we have a material that has nanoscale-related properties despite its macroscopic size. To form the network, nanoparticles are used as building blocks, giving the tremendous possibility to tailor the aerogel properties by combining different types of nanoparticles. ${ }^{10-12}$ Such a feature is extremely attractive to optimize each of the functionalities of a photocatalyst simultaneously. Furthermore, nanoparticle-based aerogels are highly porous and have a large specific surface area that makes them even more attractive as photocatalysts because the large specific surface area provides more sites where the reagents can potentially be adsorbed, while the porosity could maximize the accessibility to such adsorption sites.

Since the first nanoparticle-based aerogel was reported in $2004,{ }^{13}$ many inorganic materials of different sizes and shapes have been processed into nanoparticle-based aerogels, including semiconductors, metals, their oxides, nitrides, and fluorides and combinations thereof. ${ }^{\mathbf{1 0 1 1}}$ While the aerogels portfolio is growing, their exploration as photocatalysts remains almost unexplored. In the few existing reports, $\mathrm{TiO}_{2}, \mathrm{WO}_{x}, \mathrm{CdS}$, $\mathrm{Au}$, Pt and $\operatorname{In}_{2} \mathrm{~S}_{3}$ nanoparticles have been used as building blocks. ${ }^{7,14-19}$ Although nanoparticle-based aerogels have been tested in both liquid and gas reactions, the experimental results have indicated these materials have great potential as advanced $3 \mathrm{D}$ photocatalysts for gas phase-reactions. For instance, $\mathrm{Au}-$ 
$\mathrm{TiO}_{2}$ aerogels tested on the continuous-flow $\mathrm{CO}_{2}$ reduction reaction were highly selective towards methanol. ${ }^{15}$ Recently, we demonstrated that in comparison to their corresponding powders, the monolithic aerogels are more efficient photocatalysts for gas-phase $\mathrm{H}_{2}$ generation. ${ }^{7}$ Additionally, the study of the charge-carriers dynamics of these materials suggested that the nanoparticle network formation gives rise to shallow traps that benefit the aerogels' photocatalytic activity. ${ }^{7}$

While the results so far reported are promising, it is unclear whether the whole monolith participates in the photocatalytic reaction. Knowing this aspect is fundamental for optimizing such monolithic samples. This open question is related to the aerogel dimensions, which are way larger (at least three orders of magnitude) than the traditional photocatalysts in the form of powders and films. For instance, although films can be processed to cover a large area, their thickness is restricted to micrometers $(\mu \mathrm{m})$ to ensure their effective illumination. ${ }^{20}$ That is why when one looks at the macroscopic-sized aerogels, perhaps the first question that comes to mind is whether the incident light can reach all the nanoparticles in the aerogel structure to trigger the photocatalytic process in the whole monolith. In addition to illumination, the effectiveness of the reagent transport is another criterion that determines if the whole monolith participates in the reaction. The photocatalysis principles dictate that a particle-reagent interaction is essential for the reaction to occur. Therefore, verifying if the gas molecules diffuse through the entire monolith is also of paramount importance for these innovative 3D photocatalysts.

Furthermore, little is known about the activity of such monolithic aerogel compared to the traditional photocatalysts already reported in literature. In general, the most accepted value to compare the activity of different photocatalysts is the apparent quantum efficiency (AQE). The AQE, also known as apparent quantum yield, is calculated as the number ratio of the product(s) and the incident photons of a specific wavelength in a reaction system..$^{21-23}$ Unfortunately, so far, the activity of monolithic aerogels have been reported only in terms of reaction rates due to the design limitations of the reactors used. $^{15,24-26}$ For gas-phase reactions, typically tubular reactors are used, in which a cylindrical aerogel is placed inside of a quartz glass tube and illuminated laterally. The cylinder curvature makes it difficult to measure accurately the incident light that is essential to calculate AQE. Therefore, a reactor design that allows obtaining the AQE is needed to investigate how efficient aerogels are compared to the literature.

This work aims to address the open questions concerning light and mass transport in monolithic aerogels through optical measurements and visual experiments in anatase aerogels impregnated with organic molecules and $\mathrm{Au}$ ions. Besides, we propose a reactor design that optimizes the gas transport and the illumination of the monolith and enables the measurement of the AQE of the monolithic aerogels. Using such a robust reactor system, we performed a systematic study of the effect of the light intensity in $\mathrm{H}_{2}$ generation rate and $\mathrm{AQE}$ of the $\mathrm{Pd}$ - and $\mathrm{PdAu}$-anatase aerogels, which were used as model aerogels to assess the activity of this new class of 3D photocatalysts and compared with the traditional ones. The composition and structural features of the 3D networks of the aerogels are also presented.

\section{Results and discussion}

\section{Geometry and structural features of the aerogels}

Although the aerogels can be prepared in any geometry, we selected a disk-like shape because it can easily be illuminated from their flat sides. Two main groups of aerogels were prepared: (1) anatase aerogels with adsorbed species (dye, $\mathrm{pH}$ indicator, or metal ions) and (2) metal-anatase aerogels. Importantly, the conditions used in gelation, solvent-exchange, and drying steps of the aerogel preparation were the same to achieve similar structural features in all the aerogels. In the first group of monoliths, the anatase gels were formed and subjected to solvent-exchange before adsorbing the species mentioned above to its surface to prevent any change in their initial structural features.

Fig. 1a shows two representative digital pictures and a scanning electron microscopy (SEM) image of one of the prepared aerogels. As seen in the digital pictures, the aerogel is a translucent disk-like monolith and crack-free, which is essential to get effective mass-transport, as we explain later. The way that we performed the gelation and solvent-exchange steps played a crucial role in achieving such good-quality aerogels. For instance, the moderate gelation conditions, a combination of co-solvent (ethanol) and temperature $\left(65^{\circ} \mathrm{C}\right.$ ), allowed the nanoparticles to be assembled into a monolithic network that spans through the full volume of the initial nanoparticle dispersions, giving the advantage of controlling the monolith geometry. Besides, the ratio of water-ethanol-acetone used in the solvent-exchange step prevents the formation of cracks in the gel, keeping the monolith's integrity. Regarding the aerogel SEM study, it revealed that the microstructure of the aerogels is highly disordered and porous, as is shown in Fig. 1a. Such type of structure is characteristic of this new class of aerogels because of the nanoparticle assembly mechanism, which starts by the aggregation of small fractal clusters, which subsequently interconnect to give rise to a 3D disordered structure. ${ }^{10}$ The hysteresis in the adsorption-desorption isotherm (Fig. 1b) of the aerogels indicates the existence of mesopores (pore sizes between $2 \mathrm{~nm}$ and $50 \mathrm{~nm}$ ). The lack of a plateau in the high relative pressure region suggests the presence of macropores (pore sizes larger than $50 \mathrm{~nm}$ ). The pore size distribution curves (Fig. 1c) of the aerogels reveals a broad range of pore sizes, peaking at $31 \pm 3 \mathrm{~nm}$. Moreover, the nitrogen physisorption analysis indicated that the BET surface area of the aerogels is about $450 \mathrm{~m}^{2} \mathrm{~g}^{-1}$. In general, the aerogels' structural properties are pre-determined in the gelation step. Nevertheless, it is necessary to pay attention to the drying step, which requires being performed slowly to prevent network shrinkage, keeping the structural properties of the gel intact. In our case, we observed a volume reduction from the gel to the aerogel of only about $15 \%$.

\section{Chemical composition of the aerogels}

Although we prepared various anatase aerogels with adsorbed species, their 3D network was exclusively formed of anatase 

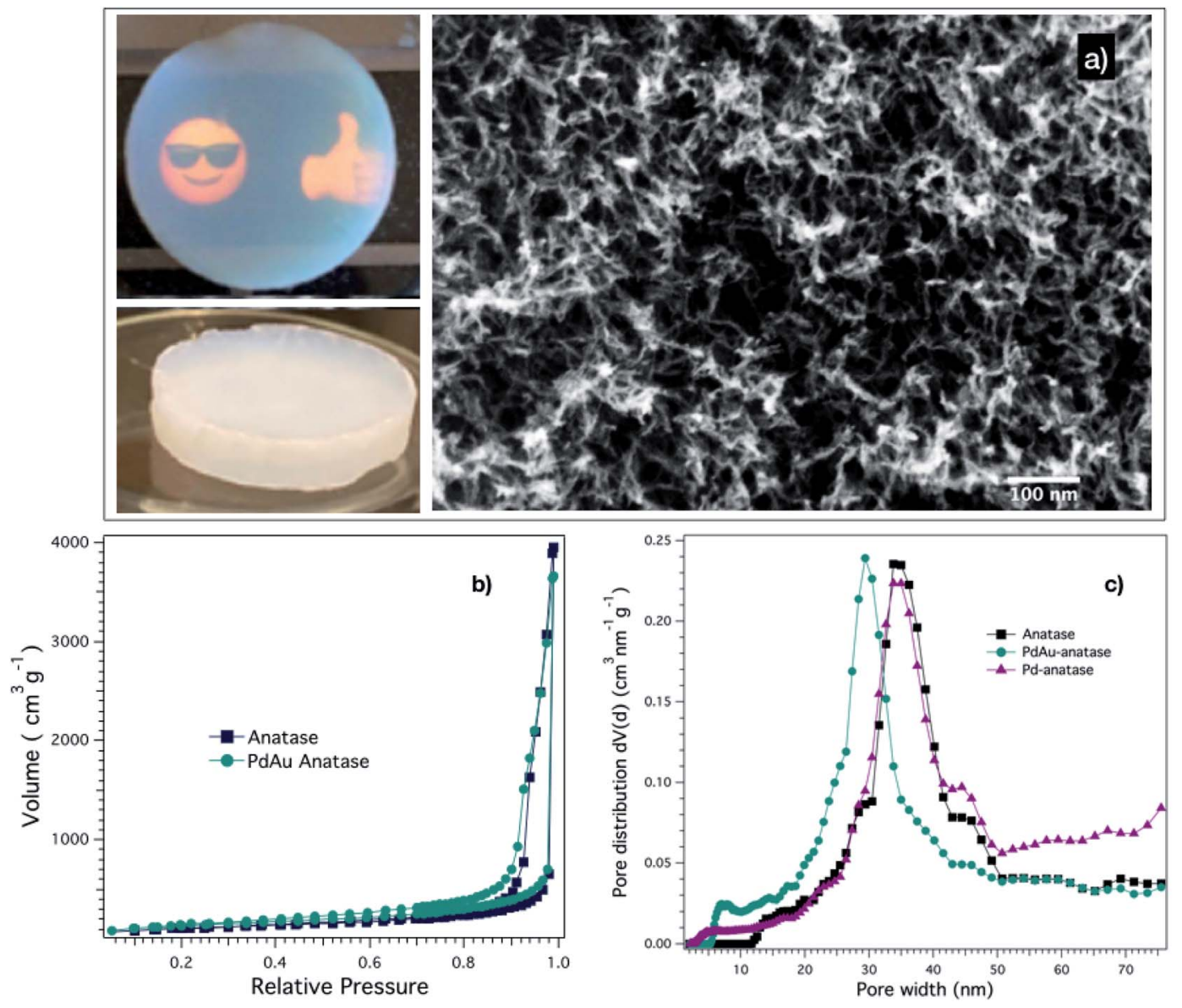

Fig. 1 The geometry of monolith aerogels and the physical characterization of their microstructure. (a) Photographs from top (above) and side (below) view of a monolithic anatase aerogel and a SEM image of its structure. (b) Nitrogen sorption isotherms and (c) pore size distribution of aerogels composed of anatase, PdAu-anatase and Pd-anatase. The nominal wt\% of metal was 0.5 in each aerogel.

nanoparticles, which was prepared following our robust reported protocol. ${ }^{14}$ We confirmed the anatase crystalline phase by XRD (Fig. S1†). Analyzing the width at half maximum of the (101) reflection employing Scherrer's equation results in anatase nanocrystal sizes of $3 \mathrm{~nm}$.

On the other hand, the metal-anatase aerogels' structures are formed by anatase and metal (Pd or PdAu) nanoparticles. The metal loading is only $0.5 \mathrm{wt} \%$. HRTEM (Fig. S2 $\dagger$ ) indicates spherical metal nanoparticles size of $3.7 \pm 2 \mathrm{~nm}$ and $3.5 \pm$ $1.3 \mathrm{~nm}$ for Pd and PdAu, respectively. The measured lattice spacing corresponds to the (111) crystal plane of $\mathrm{Pd}_{50} \mathrm{Au}_{50}$ alloy and to the plane (102) of PdO for the metal nanoparticles in the PdAu-anatase and Pd-anatase aerogels, respectively (Fig. S2 †).

\section{The importance of reactor design in the mass transport in} aerogels

In general, nanoparticle-based aerogels are expected to be 3D photocatalysts for gas-phase reactions under flow conditions. This is because the aerogel porosity is attractive to maximize the interactions of the reagents with the assembled nanoparticles. However, it is also known that despite being a highly porous material, aerogels exhibit relative low permeability due to their small pore size. ${ }^{27,28}$ Therefore, to take full advantage of the porous aerogel structure, one must control the stream of gaseous reagents in such a way that the stream goes through the monolith rather than around it. In order to achieve that, we specifically designed a photoreactor for 3D photocatalysts, see Fig. 2. The photoreactor has a reaction chamber with a quartz disk in the middle, on which the aerogel is placed. The quartz disk has four small holes in the center, see Fig. S3. $\dagger$ The idea is that the gas penetrates the aerogel from the top side, flows through the whole monolith, and leaves the aerogel at the bottom, escaping the reaction chamber through the small holes in the quartz disk.

When an aerogel of $14 \mathrm{~mm}$ diameter and $35 \mathrm{~mm}$ thickness was placed in the reactor under an argon stream of $5 \mathrm{~mL} \mathrm{~min}^{-1}$, a pressure increase of $1 \mathrm{kPa}$ in the top part of the reaction cell was observed. This was expected because of the intrinsic resistance of the aerogels to permeate the gas. ${ }^{27,28}$ So, it is essential to investigate if our reactor design leads the gas stream to go exclusively through the aerogels and the gas transport effectiveness in the entire network. To find out this, we developed an experiment, which makes it possible to visualize the pathway of the gas through the monolith with the help of a color change. We used an anatase aerogel with bromothymol blue (pH indicator) adsorbed onto its whole network. Such an aerogel was 


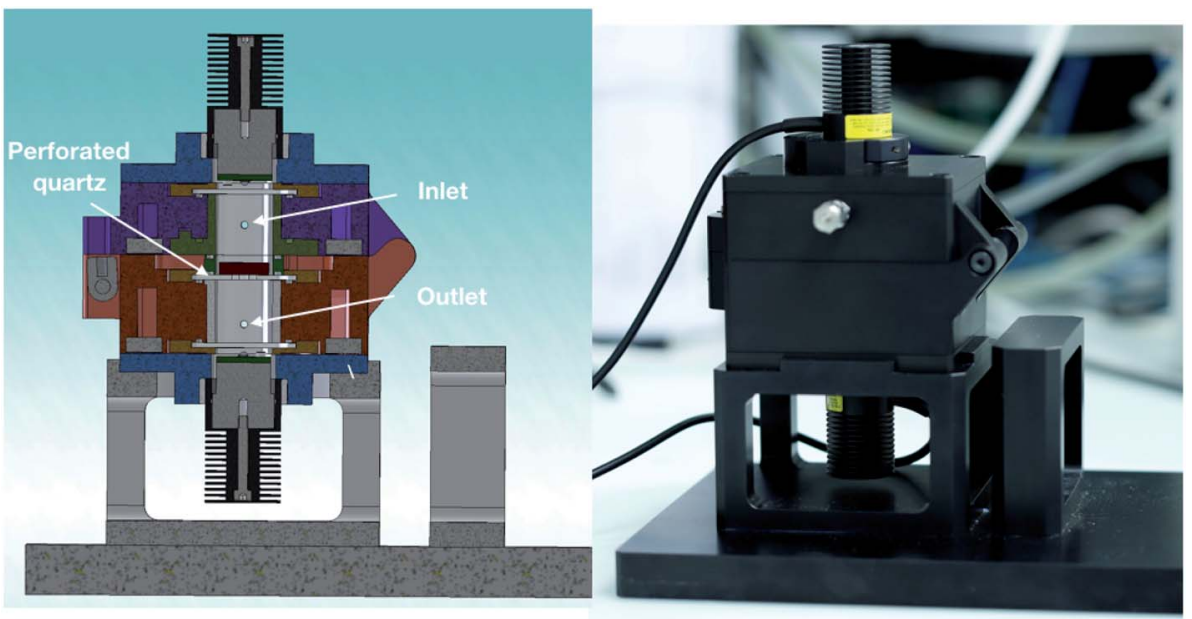

Fig. 2 Blueprint (left) and photograph (right) of the reactor designed (Tyla reactor).

placed in the reactor. A continuous flow of basic gas (ammoniasaturated argon, $5 \mathrm{~mL} \mathrm{~min}{ }^{-1}$ ) was fed into the reactor to induce a color change in the aerogel from orange to navy blue upon contact with the gas. As a result, we can see a clear indication about the direction of the gas flow. Fig. 3a shows the initial color of the anatase aerogel with adsorbed $\mathrm{pH}$ indicator. The orange color of the monolith is due to the interaction between the $\mathrm{pH}$ indicator and the acidic surface of the anatase nanoparticles. After three minutes under ammonia flow, an evident change of color in the monolith's upper and lateral surface was observed, as shown in Fig. 3b. On the other hand, four navy blue circles were observed on the aerogel's bottom side, see Fig. 3c. The blue circles correspond to the area of the aerogel that was in contact with the four exit holes in the quartz disk. The area around these circles remained orange, indicating that the gas does not leak between the aerogel and the quartz disk. In other words, our reactor makes sure that all the gas molecules pass exclusively through the monolith. Although cracks in the monolith would be a problem, our reactor design would be able to minimize the negative effect in the mass transport as long as the cracks are not located in the part of the aerogel that lies directly above one of the four exit holes. At the same time, we need to point out that cracks were not a problem in our case because our aerogels were crack-free.

According to Fig. 3b, the gas molecules entered the monolith from the side and from the top, and from there, they diffused through the monolith to reach the exit. Because of the disordered and interconnected pore structure, it is reasonable that the gas experiences a tortious diffusion in the aerogel, promoting its effective interaction with the particle network. Extending the time of ammonia flow showed that after $15 \mathrm{~min}$ the entire monolith, including the bottom part, turned navyblue (see, Fig. 3d), confirming the effective mass-transport through the entire aerogel.

The experiment with the $\mathrm{pH}$ indicator nicely showed that our reactor design promotes an effective interaction between the aerogel and the gas stream. Most importantly, we proved that the gas flow not only passes through the monolith but also diffuses through the whole 3D body, thus, ensuring that the entire aerogel network has contact with the gaseous molecular reagents during a reaction.

\section{Light transport in the monolithic aerogels}

Because the light triggers the photocatalytic process, an efficient illumination of the catalyst is essential. In principle, porous photocatalysts are promising materials for improving light-harvesting efficiency. ${ }^{29}$ Unlike the porous photocatalysts reported to date, the dimensions (particularly, the thickness) of our monolithic aerogel are much larger. This particular characteristic gives rise to the question: are the photons with suitable energy absorbed by the entire aerogel structure, or are the
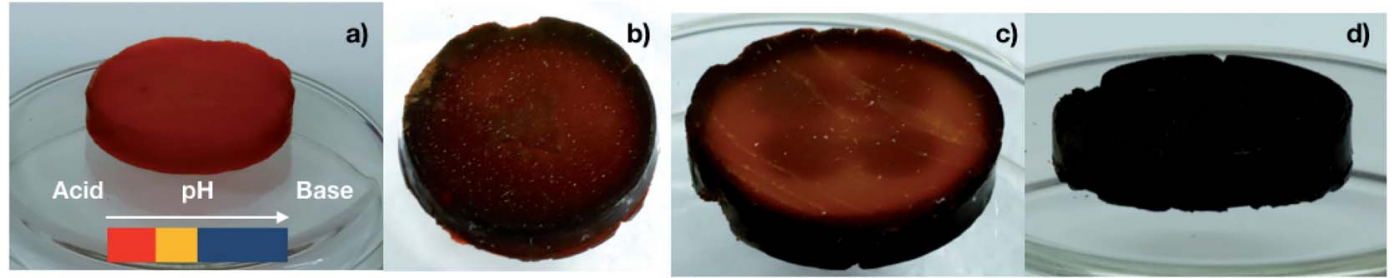

Fig. 3 Change of color of anatase aerogels with $\mathrm{pH}$ indicator under ammonia-saturated flow. The navy-blue color indicates where the gas molecules passed. (a) Initial aerogel with pH dependent color bar. (b) Top view and (c) bottom view of the aerogel monolith after 3 minutes under gas flow. (d) Aerogel after 15 minutes under gas flow. 
photons only absorbed by the nanoparticles located in or close to the aerogel surface?

To answer this question, we used anatase aerogels impregnated with methyl blue, which were illuminated from the top by a $375 \mathrm{~nm}$-LED to induce the photocatalytic degradation of the adsorbed dye. As a result of the dye removal from the surface of the anatase nanoparticles, a color change from blue to white happens, allowing us to detect the aerogel regions where the photons were absorbed. In an analogous experiment, we used surface adsorbed Au ions instead of methyl blue. In this case, the photocatalytic reaction involved the reduction of $\mathrm{Au}$ ions to Au nanoparticles, which was evidenced by the appearance of the characteristic red color of the Au nanoparticles.

To interpret the results of such experiments, one must consider how the aerogel was illuminated (related to our reactor design), the light source used, and the relative amount of the species adsorbed. Fig. 4a displays the top and the crosssectional view of a methyl blue-impregnated anatase aerogel after $18 \mathrm{~h}$ of irradiation. The top-view (inset) shows a white circle of $c a$. one centimeter diameter, indicating that the vast majority of the emitted photons impinged on this region. The illuminated area is the result of the distance between LED (quasi-point light source) and the aerogel in the reactor. In general, the larger the distance between the LED and the aerogel, the larger the illuminated area, but also the lower the light intensity as illustrated in Scheme $1 .^{30}$ The illuminated area (white circle) was achieved over time, starting as a spot in the center of the aerogels that gradually extended to reach the observed dimensions in about $4 \mathrm{~h}$. This occurred because the center of the monolithic aerogel is located directly below the LED, i.e., at the emission angle zero with respect to the LED (see, Scheme 1), where the highest intensity of the light is obtained,

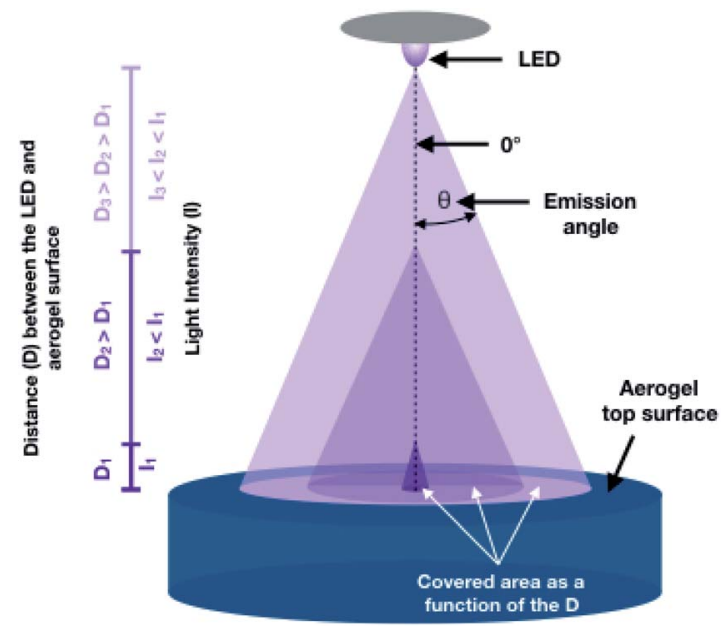

Scheme 1 Representation of the LED emission beam on the aerogel surface. The surface area of the aerogel that intercepts the cone is known as a covered area. Such area gets larger as the distance $(D)$ between the LED and the aerogel surface increases. With increasing $D$, the light intensity decreases. The emission angle is a way of describing the light spread in the conical volume. The emission zero angle $\left(0^{\circ}\right)$ is located at the center of the LED, and from it, the strongest light intensity is emitted. The intensity of the emitted light decreases at higher emission angles. In our reactor design, the aerogel center is aligned with the LED's emission angle $0^{\circ}$.

and consequently, more photons are available to initiate the photocatalytic degradation of the dye. As the emission angle of the LED increases, the light intensity decreases. Although we prolonged the irradiation time up to $18 \mathrm{~h}$, the edges of the top of the aerogel remained navy-blue, revealing that the number of incident photons in such regions (we referred to them as "dark

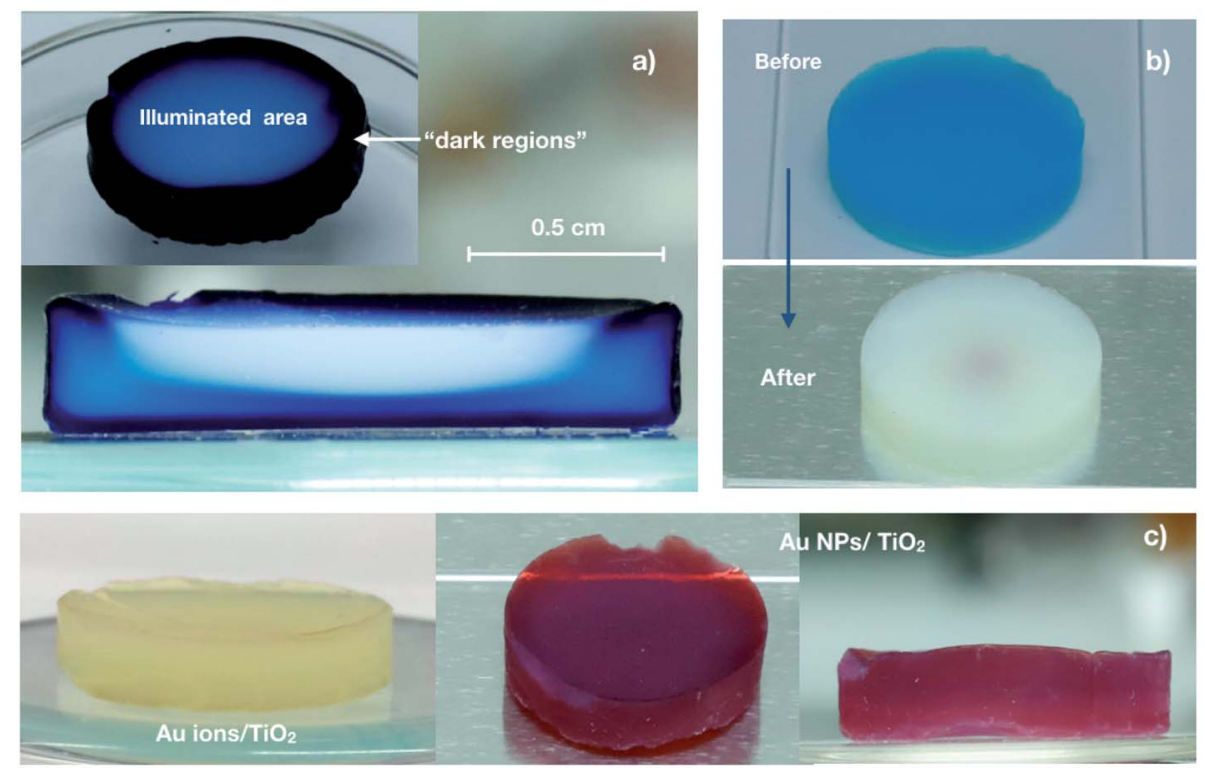

Fig. 4 Light propagation in anatase aerogels visualized by a color change caused by the photocatalytic oxidation or reduction of surface adsorbed species. (a) Cross-sectional side and top view images (inset) of an aerogel monolith impregnated with methyl blue after $18 \mathrm{~h}$ of irradiation. (b) Photograph of an anatase aerogel impregnated with methyl blue at lower dye concentration after 15 min of irradiation. (c) Photograph of an anatase aerogel impregnated with gold ions before (yellow) and after irradiation for one minute (red). 
regions") is negligible. On the other hand, the cross-sectional view of the irradiated aerogel (Fig. 4a) reveals that the dye was eliminated in the interior of the aerogel, providing clear evidence that the photons can reach the inside of the monolith. The dye degradation gives rise to a white region with a halfmoon-like shape, which coincides with the intensity distribution profile of the LED, see Fig. S4. $\dagger$ Besides the half-moon-like region, another light blue area is observed, implying that some photons diffuse through the monolith, also reaching these outer areas. Because the dye was not completely removed from the light-blue area, it is reasonable to assume that the number of photons is smaller there. Overall, this experiment with a large amount of surface adsorbed dye helps to visualize the illumination profile in the monolith, demonstrating that photons can reach the interior of the aerogel.

We also performed the experiment using an aerogel with 1000 time less dye but keeping the same irradiation conditions. Importantly, the distance between LED and the aerogel is fixed in our setup. Therefore, the illumination profile on the top and interior of the aerogel are the same as in the previous experiment. Fig. $4 \mathrm{~b}$ shows the monolith before and after one hour of irradiation. In this case, the dye was completely eliminated from the whole monolith, even from the dark regions where the illumination is negligible. It suggests that the light transport in the aerogel network is diffusive, meaning that the photons propagate randomly in the aerogel, reaching in this way even the "dark regions". As we discussed above, the number of photons decreases as they diffuse away from the photon-rich region (observed as half-moon-like shape in Fig. 4a). One can think of it as a photon diffusion gradient. Although the number of photons able to propagate long distances is relatively low, it is enough to trigger the photocatalytic degradation of methyl blue. Of course, this effect is more evident when the amount of colorant is low, as is shown in Fig. $4 \mathrm{~b}$.

The experiments using $\mathrm{Au}$ ions $/ \mathrm{TiO}_{2}$ aerogels showed similar results to those of the aerogels with adsorbed dye before described. Fig. 4c shows the photographs of an anatase aerogel with a gold content of $0.5 \mathrm{wt} \%$ before and after its irradiation. In this case, it is also observed that the red color appears in the whole monolith, suggesting again a diffusive light transport into the aerogels.

Both the experiments with the Au ions adsorbed as well as those with methyl blue suggest that the photons tend to diffuse throughout the 3D network. Generally, light diffusion is caused by scattering, which is a change of the photons' propagation direction..$^{31,32}$ To explore the diffusion characteristics of our aerogels, we performed optical measurements using laser light of $550 \mathrm{~nm}$ in wavelength. We chose the wavelength below the bandgap energy of $\mathrm{TiO}_{2}$ to decouple non-absorbing scattering from absorbing interactions, exploring in that way only the structural diffusive capabilities of the aerogels. The first simple experiment was illuminating an anatase aerogel from its bottom with a collimated beam of $\sim 1 \mathrm{~mm}$ width. The lateral view of the illuminated disk is displayed in the inset of Fig. $5 \mathrm{a}$ and shows that the entire body is illuminated. Notably, the light intensity decreases from the center of the illumination spot to
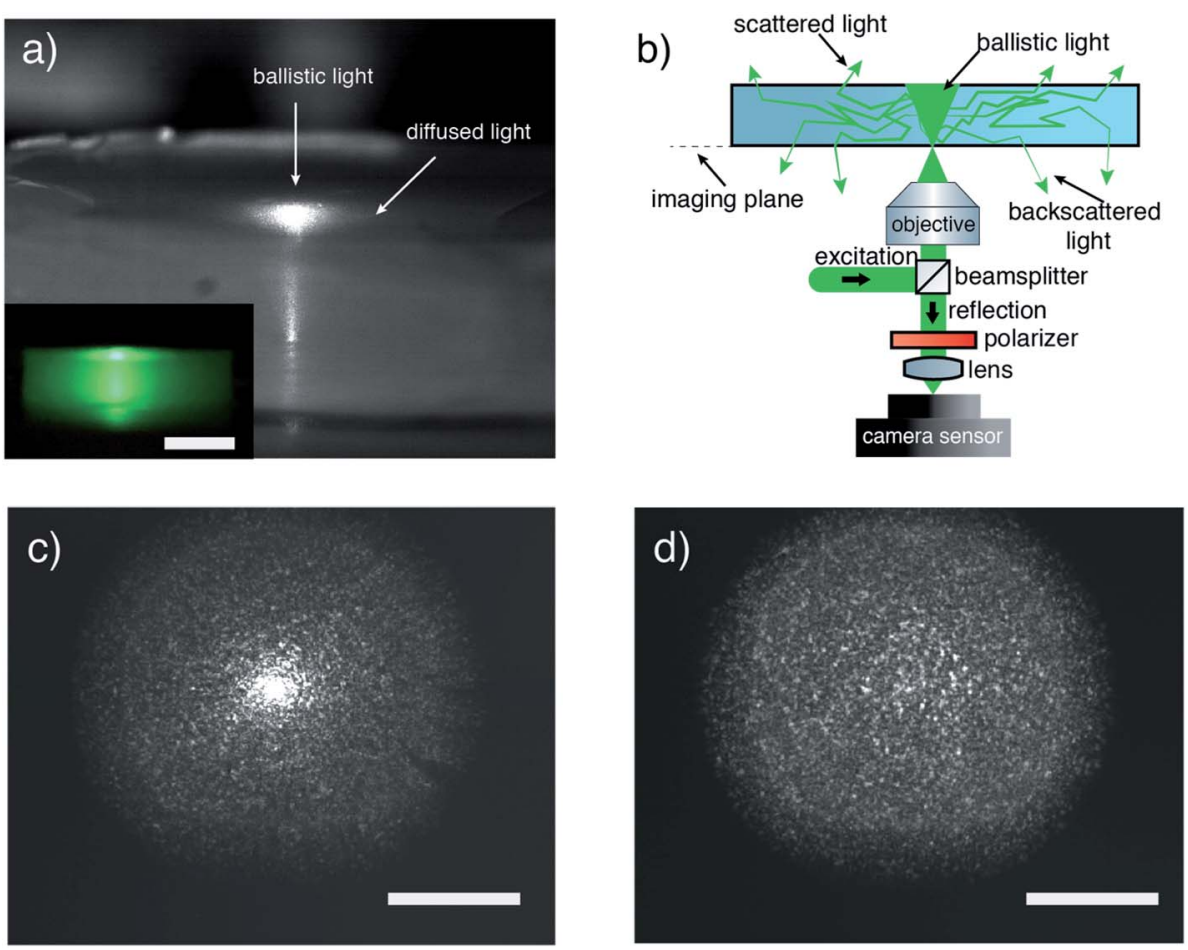

Fig. 5 Optical measurements to study the light transport using $550 \mathrm{~nm}$ laser as illumination source. (a) Telescope camera image of the aerogel illuminated from its bottom with a focused laser. (a inset) Photocamera side image of an aerogel illuminated with a collimated beam. Scale bar is $0.5 \mathrm{~cm}$. (b) Optical setup schema employed to study light transport inside the aerogel. Microscope images of the sample back-reflection (c) without and (d) with polarizer before. Scale bar is $50 \mu \mathrm{m}$. 
the edges, indicating diffusing interaction inside the aerogel. In a subsequent experiment, we use a focused laser to illuminate the aerogel bottom. An image taken with a telescope coupled CMOS camera of an illuminated aerogel is shown in Fig. 5a. It is observed that the ballistic component of the light that is scattered at the disk-air interface appears as a bright spot, while the diffused light through multiple scattering inside the body of the disk escaping from the top forms a halo around this ballistic part. To study in detail the spatial characteristics of the scattered light, we also imaged the plane of excitation (imaging plane) by collecting the reflected light following the scheme in Fig. 5b. We measured the light diffusion inside the aerogel in reflection and not in transmission to get rid of the ballistic light, which travels in forward direction and can hide the scattering effect (this is explained in more detail in the ESI $\dagger$ ). On the other hand, diffusing light has a random direction of propagation, so it is expected that we observe the same effect in forward- and back-scattering. Fig. 5c displays the backscattered light collected at the imaging plane. Two different features are observed: one concentrated bright spot in the middle and a speckle pattern around it. In this case, the bright spot indicates the part of the excitation spot reflected at the disk-air interface, showing a slightly disordered excitation spot image. Using a polarizer before the camera sensor (see, Fig. 5b), we remove the reflected spot from the image. This leads to a clean measurement of the speckle pattern that is scattered back to the objective after diffusing inside the disk (see, Fig. 5d). The speckle pattern is the result of overlapping light wavefronts with random phases. The speckle pattern clearly proves that photons traveled in random paths inside the aerogel before exiting the disk, a key characteristic of light diffusion. The broadened speckle pattern in Fig. 5c as compared to the excitation spot shows that the speckle pattern does not originate from the rough surface of the disk, but it is an effect of light diffusion inside the aerogel disk. All the above optical measurements confirm the first indications of diffusive light transport observed in the experiments with the dye and $\mathrm{Au}$ ions.

Of course, diffusion is expected to differ between different wavelengths. Diffusion relies on multiple scattering, and it is expected to increase for shorter wavelengths when the scatterers are smaller than the wavelength. ${ }^{33}$ This applies to our aerogels in which the nanoparticles are $3 \mathrm{~nm}$ in size. For this reason, we expect diffusion to be even stronger at $375 \mathrm{~nm}$ illumination. In an effort to extract characteristic values of diffusion (optical mean free path) of our aerogels, we performed time-resolved measurements. However, because of the limited temporal resolution of the optical setup we cannot extract the values confidently (details in the ESI $\dagger$ ). Despite this, the optical measurements already discussed (Fig. 5) clearly show the multiple scattering that facilitates the diffusion inside of our aerogels.

To summarize, our results deliver three important insights about light transport inside monolithic aerogels: first, light can interact with the entire monolith in a photocatalytic reaction. This is because aerogels act as a sponge, trapping the incident photons in their disordered structure. When photons enter the aerogel, they travel throughout the network increasing the probability to be absorbed by it. Second, light transport inside of the aerogels occurs by ballistic and diffusive mechanisms. And third, the diffusive transport mechanism spreads the photons through the entire network, even to the aerogel regions which were not illuminated initially.

\section{Aerogels photoactivity}

Once we confirmed experimentally that the reagent molecules and the photons diffuse through the whole monolith, we investigated the activity of the aerogels as 3D photocatalysts. For this, we used Pd- and PdAu-anatase aerogels as model samples and tested them in $\mathrm{H}_{2}$ generation reactions using methanol as a sacrificial agent. We investigated the effect of the light intensity on the photocatalytic activity of the monoliths, which were illuminated in two different ways, first from one side (top) and second from both sides (top and bottom) simultaneously to increase the number of photons reaching the structure.

Fig. 6a shows the dependence of the hydrogen evolution rate of PdAu-anatase aerogels on the light intensity. The hydrogen evolution rate increases nearly proportional $\left(I^{0.95}\right)$ with the light intensity when the aerogels were illuminated from both sides, while a more evident deviation $\left(I^{0.82}\right)$ from such a proportional relationship is observed when the monoliths were illuminated only from one side. For traditional photocatalysts, generally, the deviation from such proportionality ( $I^{a}$ when $a \neq 1$ ) is attributed to a high concentration of electron-holes pairs in the particles generated by high-intensity irradiation. ${ }^{34-36}$ In such a case, the number of electron-hole pairs is so high that their recombination is favored, reducing the overall reaction rate. Rothenberger et al. showed that the charge-carrier recombination increases obeying a second-order rate law when the average number of electron-hole pairs per particle is greater than $0.5 .^{34,35}$ Considering an irradiation of $2.3 \times$ $10^{17}$ photons per $\mathrm{s}$ (maximum irradiation achieved by illuminating the aerogel from its two sides) and the number of anatase particles in an aerogel, we calculated a rate of photon absorption per particle of $c a$. 0.2 photons per s per particle, see ESI. $\dagger$ Assuming that the charge-carrier recombination in our aerogels takes place in the nanosecond time scale as we reported previously, ${ }^{7}$ the accumulation of electron-hole pairs per nanoparticle is negligible $(\ll 0.5)$. This implies that the flux of photons $\left(2.3 \times 10^{17}\right.$ photons per $\left.\mathrm{s}\right)$ is not high enough to lead to a second-order recombination rate of the electron-hole pairs to provoke a decrease of the proportionality of the $\mathrm{H}_{2}$ rate-light intensity relationship as long as the whole nanoparticles in the monolith are illuminated homogenously. However, as we demonstrated in the experiments before, the monolith illumination is inhomogeneous because of the quasi-point spatial irradiation distribution of the LED. It means that the emitted photons impinge specific aerogel regions and from there diffuse to the rest of the network. It is likely that in such regions, the number of photons is so high that local second-order recombination is promoted, which would be a reasonable explanation for the proportionality decrease of the $\mathrm{H}_{2}$ rate-light intensity relationship. Interestingly, a higher proportionality between the light intensity and reaction rate was observed when the aerogel was illuminated from two sides (top and bottom) of the disk-like aerogel, suggesting that this way of illumination somehow helped to achieve more homogenous irradiation in the monolith. 

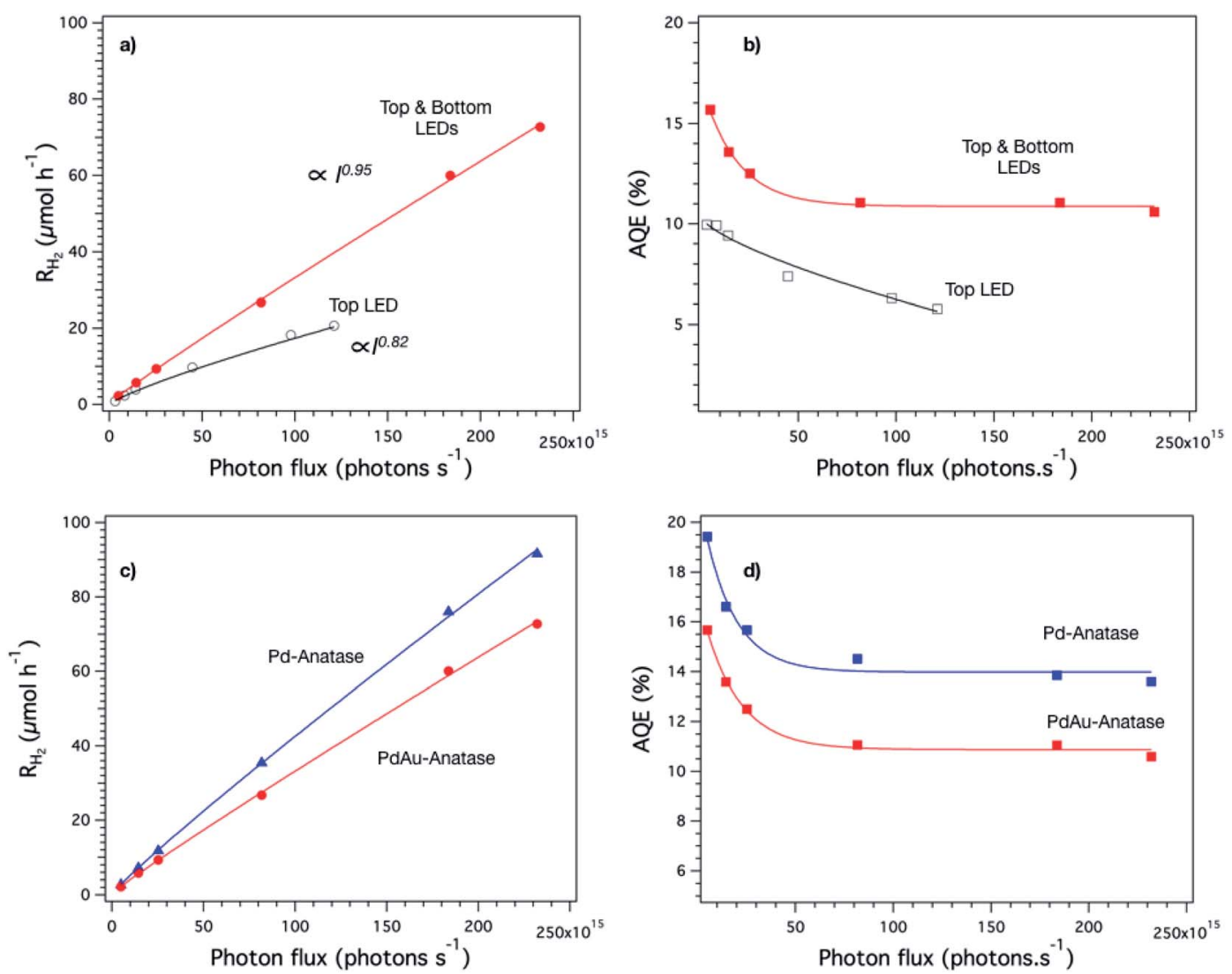

Fig. $6 \mathrm{H}_{2}$ photo-production from methanol in continuous gas-phase reaction over Pd and PdAu-anatase aerogels as a function of light intensity. (a) $\mathrm{H}_{2}$ generation rate and (b) AQE profiles of PdAu-anatase aerogels, illuminated from the top and from top \& bottom. (c) Comparison of the $\mathrm{H}_{2}$ generation rates and (d) AQE profiles of Pd- and PdAu-anatase aerogels illuminated from both sides (top \& bottom). $375 \mathrm{~nm} L E D s$ were always used as a light source.

The AQE was also influenced by the way the aerogel was illuminated as shown in Fig. 6b. When the aerogel is illuminated from both sides, the AQE $(11 \% \pm 3$ at $375 \mathrm{~nm})$ is practically independent of the light intensity. Conversely, the AQE is influenced by the light intensity when the aerogel is illuminated from one side only in agreement with its slightly lower proportionality value $\left(I^{0.82}\right)$. We performed the light intensity study also with Pd-anatase aerogels finding similar trends in both $\mathrm{H}_{2}$ reaction rates and $\mathrm{AQE}$, see Fig. S5. $\dagger$ All the above analysis showed that the evaluation of the aerogels photoactivity is not trivial. Indeed, it requires homogenous irradiation of the monoliths to allow measuring the reaction rates and AQE accurately. In this regard, the reactor design proposed in this work provides homogenous irradiation when the disk-like aerogel is illuminated from both sides, making it possible to evaluate the photocatalytic activity of aerogels.

Fig. $6 \mathrm{c}$ and $\mathrm{d}$ show a comparison of the $\mathrm{H}_{2}$ generation rate and AQE profiles as a function of the photon flux of the Pd and PdAu-anatase aerogels illuminated from two sides. In both cases, the shape of the profiles is quite similar, highlighting the robustness of the photoreactor design. However, the obtained values are different, e.g., Pd-anatase aerogels showed the highest values for $\mathrm{H}_{2}$ generation rate and AQE. This confirms that in aerogels with equal structural properties and illuminated under the same conditions, the building blocks forming the network make the difference in the photocatalytic efficiency.

To investigate the impact of the $3 \mathrm{D}$ structure in the overall activity, we pulverize the aerogels in a mortar and evaluate their

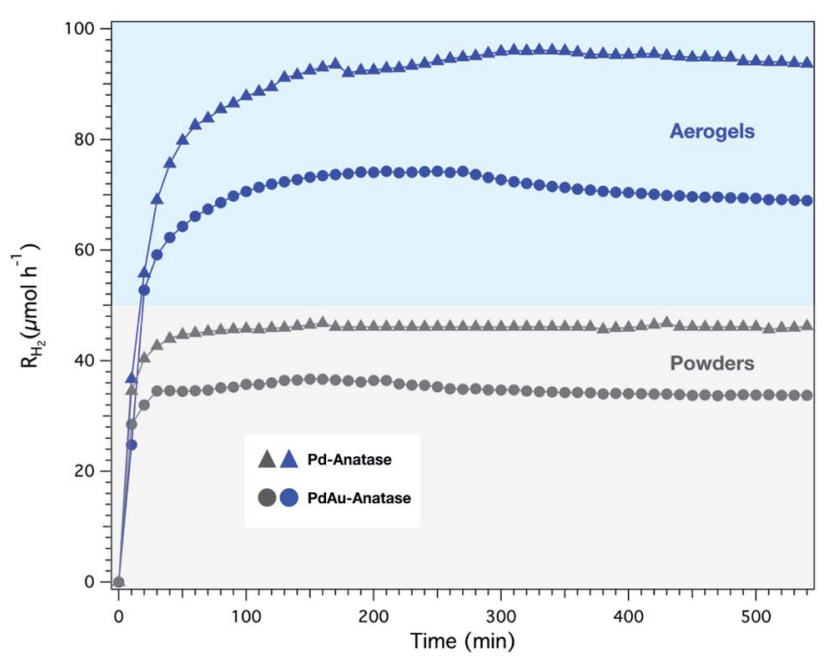

Fig. $7 \quad \mathrm{H}_{2}$ evolution rate profiles of $\mathrm{Pd}$ - and PdAu- aerogels and their respective powders. The monolith was illuminated from top and bottom with a total photon flux of $2.3 \times 10^{17}$ photons per $\mathrm{s}$. 
Table 1 Comparison of the photocatalytic activity of the monolithic aerogels with traditional (powders and films) photocatalysts for the $\mathrm{H}_{2}$ generation using methanol as electron donor

\begin{tabular}{|c|c|c|c|c|c|}
\hline Catalyst & $\mathrm{AQE}^{a}(\%)$ at $\lambda$ & $\begin{array}{l}\text { Photon flux } \\
\text { (photons per s) }\end{array}$ & $\begin{array}{l}\text { Measured rate } \\
\left(\mu \mathrm{mol} \mathrm{h}{ }^{-1}\right)\end{array}$ & $\begin{array}{l}\text { Reaction } \\
\text { phase }\end{array}$ & Reference \\
\hline $0.5 \mathrm{wt} \% \mathrm{Pd}$-anatase & 13 at $375 \mathrm{~nm}$ & $2.3 \times 10^{17}$ & 92 & Gas & This work \\
\hline 0.5 wt $\%$ PdAu-anatase & 11 at $375 \mathrm{~nm}$ & $2.3 \times 10^{17}$ & 73 & Gas & This work \\
\hline $\begin{array}{l}0.7 \mathrm{wt} \% \mathrm{Pt} / \mathrm{TiO}_{2} / \mathrm{SiO}_{2} / \mathrm{Si} \text { (supported } \\
\text { powders) }\end{array}$ & $\sim 2$ at $365 \mathrm{~nm}$ & $1.4 \times 10^{17}$ & 4.2 & Gas & 40 \\
\hline $1.5 \mathrm{wt} \%$ Pt-anatase (supported powders) & 0.2 at $330-400 \mathrm{~nm}$ & $2 \times 10^{18 b}$ & 4 & Gas & 41 \\
\hline $1 \mathrm{wt} \% \mathrm{Au}-\mathrm{TiO}_{2}$ (supported powders) & 6.3 at $330-400 \mathrm{~nm}$ & $7.1 \times 10^{17 b}$ & 142 & Gas & 42 \\
\hline $0.2 \mathrm{wt} \% \mathrm{Pt}-\mathrm{TiO}_{2}(\mathrm{P} 25$, powders $)$ & 3.6 at $365 \mathrm{~nm}$ & $7.4 \times 10^{17 b}$ & 80 & Liquid & 39 \\
\hline $\begin{array}{l}0.2 \mathrm{wt} \% \mathrm{Pt}-\mathrm{TiO}_{2} \text { (mesoporous anatase, } \\
\text { powders) }\end{array}$ & 10.7 at 365 & $7.4 \times 10^{17 b}$ & 237 & Liquid & 39 \\
\hline $2.5 \mathrm{wt} \% \mathrm{Pd}-\mathrm{TiO}_{2}(\mathrm{P} 25$, powders $)$ & 12 at $365 \mathrm{~nm}$ & $4 \times 10^{17}$ & 170 & Liquid & 43 \\
\hline${ }^{a} \mathrm{AQE}$ refers to apparent quantum efficier & calculated using the & ving equation: $\mathrm{A}$ & $\frac{2 n\left(\mathrm{H}_{2}\right)}{n \text { (incident phot }}$ & \multicolumn{2}{|c|}{${ }^{b}$ Calculated value from the } \\
\hline
\end{tabular}

activity. Fig. 7 displays the $\mathrm{H}_{2}$ evolution rates over time of the aerogels and their corresponding powders. Clearly, the aerogel structure favors the $\mathrm{H}_{2}$ evolution, delivering an efficiency two times higher than the powders independently of the aerogel composition.

Because it is the first time that AQE of nanoparticle-based aerogels is reported, we compared their AQE and reaction rates with photocatalysts with similar compositions already reported in the literature (see Table 1). Notice that the reported works were carried out using UV-light sources with a peak wavelength at $365 \mathrm{~nm}$, which is shorter than in our case $(375 \mathrm{~nm})$. It is important to point this out because it has been demonstrated that the AQE decreases as the energy of the irradiation wavelengths approaches the absorption edge of $\mathrm{TiO}_{2}$ nanoparticles. ${ }^{37,38}$ Although our aerogels were irradiated with lower-energy photons, their AQEs were notably higher than that of the traditional photocatalysts used in gas-phase reactions, as is shown in Table 1 . In both aerogels and the references, crystalline anatase nanoparticles were used as semiconductors. Therefore, the co-catalyst and the $3 \mathrm{D}$ porous structure were the main differences between the aerogels and the references. So far, platinum (Pt) and $\mathrm{Au}$ are the only two metals reported as cocatalysts for gas-phase $\mathrm{H}_{2}$ evolution reactions. Nevertheless, the comparison is fair because $\mathrm{Pt}$ is considered the most efficient cocatalyst for $\mathrm{H}_{2}$ generation. ${ }^{3}$ Although with $\mathrm{Pd}$ we used a less efficient metal as co-catalysts, the aerogel AQE values were up to 65 times higher. This suggests that the $3 \mathrm{D}$ porous structure of the aerogels offers a tremendous advantage as photocatalyst, giving access to the reagents to interact with the nanoparticles and maximizing the light-harvesting, as we demonstrated above.

We also compared the AQE of the aerogels obtained in gasphase reactions with those of the traditional photocatalysts evaluated in liquid-phase reactions (powder dispersions), see Table 1 . The study by Bahnemann's group is the most comparable with our aerogels because they investigated the performance of mesoporous $\mathrm{Pt}-\mathrm{TiO}_{2}$ powders. ${ }^{39}$ The AQE achieved by such powders was 10.7, which is, for example, $17 \%$ lower than our $\mathrm{Pd}-\mathrm{TiO}_{2}$ aerogel. It shows that by using aerogels as photocatalysts, the $\mathrm{H}_{2}$ evolution reaction performed in the gas-phase is more efficient than in liquid-phase. Again, this is due to the structural advantages of the aerogels acting as photon and reagent sponge, and their pores function as confined reaction spaces favorable for the reaction to occur efficiently. It is also important to mention that the volume of our aerogels was 0.5 $\mathrm{cm}^{3}$, which is very small compared to the volume of the powder dispersions. For example, in the evaluation of the mesoporous $\mathrm{Pt}-\mathrm{TiO}_{2}$ powders, Bahnemann et al. used a volume reaction of $75 \mathrm{~mL}$, which is 150 -fold larger than in our case. It is important from the practical application point of view because instead of having large reaction pools, a compact and efficient monolithic aerogel can be used as a photocatalytic system.

\section{Conclusion}

It was shown that light travels inside metal-anatase aerogels in diffusive and ballistic ways due to their disordered porous structure. The diffusive light transport spreads the photons all over the 3D network, even to the aerogel regions, which were not illuminated initially. It results in an entire photoactive monolith despite its large dimensions for a photocatalyst. Similarly, gas molecules can effectively diffuse in the entire network as long as the used reactor ensures that the gas stream passes exclusively through the monolith as in our photoreactor.

It was also proven that our reactor design, Tyla, is a robust and practical system that can be taken as a reactor reference to evaluate monolithic 3D photocatalysts. With Tyla, it was measured the AQE of monolithic nanoparticle-based aerogels for the first time using Pd- and PdAu-anatase as model aerogels. The aerogels activity analysis pointed out four main aspects: first, the aerogels' activity amazingly exceeded (up to 65 times) that of the traditional catalysts already reported for gas flow reactions. Second, Pd- and PdAu-anatase were two times more active than their crushed powders. Such difference in activity was the same because the aerogels had the same structural features. Third, between aerogels with similar structure, the building blocks determines the aerogel performance. For instance Pd-anatase aerogels were more active than PdAu- 
anatase ones. Fourth, compact aerogels are as good or more efficient catalysts as traditional volume-demanding dispersions already reported.

\section{Experimental section}

\section{Chemicals}

Titanium(Iv) tetrachloride (99.9\% trace metals basis), benzyl alcohol (puriss., 99-100.5\% (GC)), 2-amino-2-(hydroxymethyl)1,3-propanediol (Trizma ${ }^{\circledR}$ base, puriss., $\geq 99.7 \%$ ), chloroform ( $\geq 99.8 \%$ ), diethyl ether (for HPLC, $\geq 99.9 \%$, inhibitor-free), ethanol (absolute), methanol (analytic grade), potassium tetrachloropalladate(II) (98\%), acetone (for HPLC $\geq 99.8 \%$ ), ammonium hydroxide solution $28 \% \mathrm{NH}_{3}$ in $\mathrm{H}_{2} \mathrm{O}$, and bromothymol blue were purchased from Sigma-Aldrich. Gold(III) chloride trihydrate (99\%) was purchased from Acros. Sodium borohydride and methyl blue was purchased from VWR chemicals and Fluka, respectively. Liquid carbon dioxide $(\geq 99 \%)$ and argon (99.999\%) were provided by PanGas AG, Switzerland. All chemicals were used as received without further purification.

\section{Nanoparticle synthesis and dispersion preparation}

Anatase nanoparticles were synthetized by non-aqueous sol-gel route and redispersed in water following our reported protocol. ${ }^{44}$ Briefly, $414 \mathrm{mg}$ of Trizma ${ }^{\circledR}$ were dissolved in $90 \mathrm{~mL}$ benzyl alcohol at $80{ }^{\circ} \mathrm{C}$ in an oil bath. After cooling it to room temperature, $4.8 \mathrm{~mL}$ of $\mathrm{TiCl}_{4}$ were added under vigorous stirring. The reaction solution was heated again to $80^{\circ} \mathrm{C}$ and stirred for $24 \mathrm{~h}$. The white precipitate was separated from the solution by centrifugation and washed three times with chloroform and subsequently three times with diethyl ether. Finally, the wet powder was dispersed in $30 \mathrm{~mL}$ of deionized water, resulting in a highly stable $\mathrm{TiO}_{2}$ dispersion with a concentration of $90 \mathrm{~g} \mathrm{~L}^{-1}$. The metal nanoparticles ( $\mathrm{Pd}$ and $\mathrm{PdAu}$ ) were synthesized directly in the anatase dispersions. Shortly, $6 \mathrm{~mL}$ of the anatase dispersion were transferred to a vial. Two vials were prepared. Then, the corresponding amount of metal precursor(s) to have a total metal loading of $0.5 \mathrm{wt} \%$ was added to each vial. For Pd nanoparticles, $8.6 \mathrm{mg}$ of $\mathrm{K}_{2} \mathrm{PdCl}_{4}$ was added to the dispersion. For PdAu nanoparticles, 3 and $3.6 \mathrm{mg}$ of $\mathrm{K}_{2} \mathrm{PdCl}_{4}$ and $\mathrm{HAuCl}_{4}$ were added to the $6 \mathrm{~mL} \mathrm{TiO}_{2}$ dispersion. Once the metal precursors were dissolved, $5.2 \mathrm{~mL}$ of ethanol were added to each dispersion. The vials were capped, stirred for 15 min and degassed with $\mathrm{N}_{2}$. The metal ions were reduced by injecting quickly $0.8 \mathrm{~mL}$ of $\mathrm{NaBH}_{4}$ ethanolic solution to the vigorously stirred dispersions. The concentrations of the injected $\mathrm{NaBH}_{4}$ solutions were 83 , and $57 \mathrm{mM}$ for Pd, and $\mathrm{PdAu}$, respectively. The two dispersions were kept under stirring for $30 \mathrm{~min}$. The concentration of Pd- and PdAu-anatase dispersions was $45 \mathrm{~g} \mathrm{~L}^{-1}$.

\section{Aerogel preparation}

The three prepared dispersions were used to form their corresponding 3D networks. For anatase gels, anatase dispersion was diluted at $1: 1(\mathrm{v} / \mathrm{v})$ with $57 \mathrm{mM} \mathrm{NaBH}_{4}$ ethanolic solution. After, $1.5 \mathrm{~mL}$ was transferred to a $20 \mathrm{~mL}$ syringe, which was the gelation mould. Then, the syringe was capped and heated in an oven at $65{ }^{\circ} \mathrm{C}$ for $c a .20 \mathrm{~min}$, obtaining a gel. The procedure was the same for Pd- and PdAu-anatase dispersions. Nevertheless, for such dispersions, it was not necessary to dilute them because the nanoparticles were already dispersed in $1: 1(\mathrm{v} / \mathrm{v})$ ethanol-water. The obtained gels were subjected to solventexchange. For this, each gel was immersed into $10 \mathrm{~mL}$ of a water/ethanol/acetone $=10 / 20 / 70 \mathrm{v} / \mathrm{v}$ mixture. They were then transferred to $10 \mathrm{~mL}$ of a water/ethanol/acetone $=5 / 15 / 80 \mathrm{v} / \mathrm{v}$ mixture, and finally to the same volume of dry acetone. The time between each solvent exchange was six hours.

Once the solvent-exchange was completed, bromothymol blue ( $\mathrm{pH}$ indicator), methyl blue, or gold ions were adsorbed to whole network anatase gels. For this, each of the anatase aerogels was immersed into $7 \mathrm{~mL}$ of an acetonic solution of the corresponding species. For bromothymol blue and gold ions, the concentration of the solution was $5 \times 10^{-5} \mathrm{mM}$ and $6 \times$ $10^{-5} \mathrm{mM}$, respectively. For methyl blue, 250 and $0.25 \mathrm{mg} \mathrm{L}^{-1}$ were the two concentrations used. The anatase gels were immersed in such solutions for $24 \mathrm{~h}$ and subsequently dried. The step above was skipped for the metal-anatase gels, which were directly dried after the solvent-exchange step.

To dry the gels, they were placed into the chamber of a critical point dryer (E3100, Quorum) set at $10{ }^{\circ} \mathrm{C}$. The chamber was filled with liquid $\mathrm{CO}_{2}$. After, around a quarter of the $\mathrm{CO}_{2}$ volume was drained from the chamber, taking care to keep the gels always immersed to avoid the formation of cracks in the monoliths. The empty volume was filled again with liquid $\mathrm{CO}_{2}$. This procedure was repeated every fifteen minutes for one hour. Afterward, the temperature was raised to $40{ }^{\circ} \mathrm{C}$ to reach supercritical conditions, which were kept for one hour. Finally, the system was depressurized slowly, resulting in aerogel monoliths with minimal shrinkage. The three main groups of prepared aerogels were the following: (1) anatase, (2) anatase with adsorbed species ( $\mathrm{pH}$ indicator, dye, or $\mathrm{Au}$ ions), and (3) metal (Pd or PdAu)-anatase aerogels.

\section{Characterization}

Scanning electron microscopy (SEM) images were recorded on a Zeiss Leo-1530. Prior to SEM analysis, the samples were coated with $6 \mathrm{~nm}$ Pt. High-resolution transmission electron microscopy (HRTEM) in both TEM and scanning (STEM) modes were performed on a FEI Talos F200X operated at $200 \mathrm{kV}$. Powder Xray diffraction (XRD) measurements were performed on a PANalytical Empyrean equipped with a PIXcel 1D detector and $\mathrm{Cu}$ $\mathrm{K} \alpha \mathrm{X}$-ray irradiation. Nitrogen gas sorption analysis was carried out on a Quantachrome Autosorb iQ at $77 \mathrm{~K}$. Prior to the measurements, the samples were outgassed at $100{ }^{\circ} \mathrm{C}$ for $24 \mathrm{~h}$. The surface area was determined via the BET method, and the pore size distribution and total pore volume were calculated by a DFT analysis using a nonlocal DFT calculation model for nitrogen at $77 \mathrm{~K}$ based on cylindrical pores in silica.

\section{Photocatalytic reactor design for monolithic photocatalysts}

To perform the studies of light and mass transport as well as the photocatalytic activity of the 3D monolithic aerogels, a dedicated reactor was designed. Fig. 2 shows a cross-section view of the reactor. The internal aluminium reaction cell is a two-parts 
cylindrical chamber in a vertical position with open ports at the top and bottom to attach quartz pieces through which the reaction cell is illuminated. The quartz optical windows are flat to avoid distortion of the incident light, ensuring most of the light to pass through. The quartzes are fitted to the reaction cell by sandwiching them with a holder plate and a nitrile butadiene rubber (NBR) O-ring to ensure gas tightness. The light sources, $375 \mathrm{~nm}$ high-power light emitting diodes (LEDs, Thorlabs), were placed $2 \mathrm{~mm}$ apart of the quartz windows. The LEDs are easily attached to the reactor by a slip ring (SM1RC, Thorlabs) and controlled by analogous drivers (LEDD1B, Thorlabs). The sample holder is placed in the middle of the reaction cell so that the aerogel is at the same distance $(27.5 \mathrm{~mm})$ between the top and bottom light source. In the center of the sample holder, four holes of $2 \mathrm{~mm}$ diameter are presented. The two parts of the reaction cell are joined by a hinge, which permits opening the cell by folding the upper part (in purple) 180 degrees and placing the aerogel monolith in the holder easily. The gaseous reagents are fed through the inlet located at the upper chamber (in purple) while the outlet is at the bottom.

\section{Mass and light transport in monolithic aerogels}

Mass transport. For this study, the anatase aerogels with adsorbed bromothymol blue were used as a sample. The aerogel was placed in the reactor. $5 \mathrm{~mL} \mathrm{~min}^{-1}$ of argon saturated with ammonia were fed to the reactor by bubbling the argon flow through a reservoir containing $20 \mathrm{mM}$ aqueous ammonia solution. The flow was kept only for three minutes. After that, the visible change in color of the aerogels was recorded by taking digital pictures. The experiment was performed three times under three-minutes flow, and the other three ones extending the flow time to 15 minutes.

Light transport. Anatase with adsorbed methyl blue was placed in the reactor. An air flow $\left(5 \mathrm{~mL} \mathrm{~min}^{-1}\right)$ was feed to the reactor. Then, the top LED was turned on. The incident photon flux was $1.2 \times 10^{17}$ photons per s. The irradiation time was either $18 \mathrm{~h}$ or one hour. The resulting change of color (from blue to white) in the aerogels was documented by taking digital images of the aerogel after irradiation. The light transport study was also performed using anatase aerogels with $\mathrm{Au}$ ions adsorbed as samples. The experimental protocol was similar to the above, but in this case, a $5 \mathrm{~mL} \mathrm{~min}^{-1}$ flow of argon saturated with methanol/water was fed to the system instead of air. The aerogels were illuminated for less than $3 \mathrm{~min}$. Photographs were taken of the aerogels after their illumination.

Optical measurements were performed on the disk-shaped aerogels to further investigate the light transport inside the monoliths using the monochromatic light of a polarised laser excitation at $550 \mathrm{~nm}$ and an objective of $0.8 \mathrm{NA}$ at an inverted microscope configuration. The collimated excitation laser light is coupled through a $50: 50$ beam splitter inside the objective and focused on the monolith's bottom. The reflection collected by the same objective is then decoupled by the excitation through the beam splitter and is then focused through a $125 \mathrm{~mm}$ focal distance lens onto a CMOS DCC1645C camera sensor by Thorlabs with $1280 \times 1024$ pixels and pixel size of 5.2 $\mu \mathrm{m}$. For the measurement of Fig. $5 \mathrm{~d}$ the reflected light is filtered by a polarizer oriented in a way to reject the excitation polarization before being focused on the camera sensor. For the image of Fig. 5a inset, the objective was removed to let the collimated beam interact with the sample. The telescope image in Fig. $5 \mathrm{c}$ is taken in ambient light conditions to show the disk structure along with the laser light patterns.

\section{Photocatalytic performance evaluations}

We investigated the photoactivity of the monolithic aerogels using Pd- and PdAu aerogels as model samples. The target reaction was the gas-phase $\mathrm{H}_{2}$ generation, carried out in continuous flow and using methanol as an electron donor. The aerogel was placed in the reaction chamber. Then, $5 \mathrm{~mL} \mathrm{\textrm {min } ^ { - 1 }}$ of argon saturated with methanol-water, which was obtained by bubbling the argon flow through a reservoir filled with a 50 vol\% methanol-water solution at room temperature was fed to the reactor. Before the irradiation, the air in the reactor chamber was evacuated over time by the reagent flow. The oxygen levels were analyzed at the reactor outlet with a gas chromatograph (Micro-GC 3000A with thermal conductivity detector, He and Ar carrier gases). When the oxygen concentration was $<50 \mathrm{ppm}$, the reaction was launched by turning the $\operatorname{LED}(\mathrm{s})$ on. According to the analysis, the aerogel was illuminated with one or two LEDs. The emitted light intensity was analogously controlled. And the incident irradiation was measured by a thermal power sensor ( $5 \mathrm{~W}$, Thorlabs). The evolved $\mathrm{H}_{2}$ was analyzed by a gas chromatograph taking automatically a sample every $10 \mathrm{~min}$. The $\mathrm{H}_{2}$ production rates were calculated from the $\mathrm{H}_{2}$ concentration obtained at $180 \mathrm{~min}$ (time needed for the reaction to reach the steady-state). After $180 \mathrm{~h}$, the illumination was turned off. The reagent flow was increased from 5 to $40 \mathrm{~mL} \mathrm{~min}^{-1}$ to remove quickly the $\mathrm{H}_{2}$ that remained in the system. After approx. 15 minutes, the reagent flow was reduced again to $5 \mathrm{~mL} \mathrm{~min}^{-1}$. The flow at the outlet of the reactor was analyzed by gas chromatography to confirm the absence of $\mathrm{H}_{2}$ or $\mathrm{O}_{2}$. Once it was achieved, the sample was illuminated again with higher irradiation. For each sample, six different intensity values were used (see, Table $S 1 \dagger$ ).

In addition to the light intensity study, we also evaluated aerogel activity for a longer time $(9 \mathrm{~h})$. In this case, the aerogel was illuminated by the top and bottom LEDs using a total photon flux of $2.3 \times 10^{17}$. The flow rate and the sampling time were also $5 \mathrm{~mL} \mathrm{~min}^{-1}$ and $10 \mathrm{~min}$, respectively. To compare the activity of the aerogel with their respective powders, we pulverized the corresponding aerogels. The sample holder (quartz with four holes) was exchanged by quartz without holes. The powders were placed on an area of $c a .1 .5 \mathrm{~cm}^{2}$, which was the same area covered by the monoliths. The powders were also illuminated by both top and bottom LEDs. The rest of the conditions were the same that the above mentioned.

\section{Conflicts of interest}

There are no conflicts to declare. 


\section{Note after first publication}

'Photocatalytic reactor design for monolithic photocatalysts', the distance between the aerogel and the top and bottom light source has been corrected from $2.75 \mathrm{~mm}$ (incorrect value given in the first publication) to $27.5 \mathrm{~mm}$.

\section{Acknowledgements}

Financial support by ETH Zürich is gratefully acknowledged. The authors also thank the Scientific Center for Optical and Electron Microscopy (ScopeM) of ETH Zurich for providing the electron microscopy facilities.

\section{References}

1 Y. He and D. Wang, Chem, 2018, 4, 405-408.

2 B. A. Pinaud, J. D. Benck, L. C. Seitz, A. J. Forman, Z. Chen, T. G. Deutsch, B. D. James, K. N. Baum, G. N. Baum, S. Ardo, H. Wang, E. Miller and T. F. Jaramillo, Energy Environ. Sci., 2013, 6, 1983-2002.

3 Q. Wang and K. Domen, Chem. Rev., 2020, 120, 919-985.

4 S. Linic, P. Christopher and D. B. Ingram, Nat. Mater., 2011, 10, 911-921.

5 R. Van De Krol and M. Grätzel, Photoelectrochemical Hydrogen Production, Springer, US, Boston, MA, 2012, vol. 102.

6 M. Schreck and M. Niederberger, Chem. Mater., 2019, 31, 597-618.

7 A. L. Luna, F. Matter, M. Schreck, J. Wohlwend, E. Tervoort, C. Colbeau-Justin and M. Niederberger, Appl. Catal., B, 2020, 267, 118660.

8 W. Wan, R. Zhang, M. Ma and Y. Zhou, J. Mater. Chem. A, 2018, 6, 754-775.

9 S. Alwin and X. Sahaya Shajan, Materials for renewable and sustainable energy, 2020, 9, 1-27.

10 F. Matter, A. L. Luna and M. Niederberger, Nano Today, 2020, 30, 100827.

11 C. Ziegler, A. Wolf, W. Liu, A.-K. Herrmann, N. Gaponik and A. Eychmüller, Angew. Chem., Int. Ed., 2017, 56, 13200-13221.

12 F. Rechberger and M. Niederberger, Nanoscale Horiz., 2017, 2, 6-30.

13 J. L. Mohanan and S. L. Brock, J. Non-Cryst. Solids, 2004, 350, 1-8. 14 F. J. Heiligtag, M. D. Rossell, M. J. Süess and M. Niederberger, J. Mater. Chem., 2011, 21, 16893-16899.

15 F. Rechberger and M. Niederberger, Mater. Horiz., 2017, 4, 1115-1121.

16 T. Berestok, P. Guardia, J. B. Portals, S. Estradé, J. Llorca, F. Peiró, A. Cabot and S. L. Brock, Langmuir, 2018, 34, 6470-6479.

17 L. Korala, J. R. Germain, E. Chen, I. R. Pala, D. Li and S. L. Brock, Inorg. Chem. Front., 2017, 4, 1451-1457.

18 M. Niederberger, R. O. da Silva, H. Junge, M. Karnahl, S. Wohlrab and F. J. Heiligtag, Catal. Today, 2014, 246, 101-107.

19 F. J. Heiligtag, W. Cheng, V. R. de Mendonça, M. J. Süess, K. Hametner, D. Günther, C. Ribeiro and M. Niederberger, Chem. Mater., 2014, 26, 5576-5584.
20 T. Van Gerven, G. Mul, J. Moulijn and A. Stankiewicz, Chem. Eng. Process., 2007, 46, 781-789.

21 B. Ohtani, J. Photochem. Photobiol., C, 2010, 11, 157-178.

22 H. Kisch and D. Bahnemann, J. Phys. Chem. Lett., 2015, 6, 1907-1910.

23 M. Qureshi and K. Takanabe, Chem. Mater., 2017, 29, 158167.

24 A. Jonoidi Jafari, M. Kermani, A. Hosseini-Bandegharaei, A. Rastegar, M. Gholami, A. Alahabadi and G. Farzi, Chem. Eng. Res. Des., 2019, 150, 1-13.

25 H. Wang, G. Wang, Y. Zhang, Y. Ma, Z. Wu, D. Gao, R. Yang, B. Wang, X. Qi and J. Yang, Sci. Rep., 2019, 9, 16314.

26 J. Yang, Q. Shi, R. Zhang, M. Xie, X. Jiang, F. Wang, X. Cheng and W. Han, Carbon, 2018, 138, 118-124.

27 P. H. Dieudonne and J. Phalippou, J. Sol-Gel Sci. Technol., 1999, 14, 249-256.

28 B. Hosticka, P. M. Norris, J. S. Brenizer and C. E. Daitch, J. Non-Cryst. Solids, 1998, 225, 293-297.

29 X. Li, J. Yu and M. Jaroniec, ChemInform, 2016, 47, 2603-2636.

30 C. Casado, R. Timmers, A. Sergejevs, C. T. Clarke, D. W. E. Allsopp, C. R. Bowen, R. van Grieken and J. Marugán, Chem. Eng. J., 2017, 327, 1043-1055.

31 K. Fujita, J. Konishi, K. Nakanishi and K. Hirao, Sci. Technol. Adv. Mater., 2006, 7, 511-518.

32 D. S. Wiersma, A. Muzzi, M. Colocci and R. Righini, Phys. Rev. E: Stat. Phys., Plasmas, Fluids, Relat. Interdiscip. Top., 2000, 62, 6681-6687.

33 E. Hecht, Optics, Addison-Wesley, Reading, Mass, 4th edn, (C)2002.

34 T. Hisatomi, K. Maeda, K. Takanabe, J. Kubota and K. Domen, J. Phys. Chem. C, 2009, 113, 21458-21466.

35 G. Rothenberger, J. Moser, M. Gratzel, N. Serpone and D. K. Sharma, J. Am. Chem. Soc., 1985, 107, 8054-8059.

36 C. Colbeau-Justin, M. Kunst and D. Huguenin, J. Mater. Sci., 2003, 38, 2429-2437.

37 A. L. Luna, E. Novoseltceva, E. Louarn, P. Beaunier, E. Kowalska, B. Ohtani, M. A. Valenzuela, H. Remita and C. Colbeau-Justin, Appl. Catal., B, 2016, 191, 18-28.

38 A. L. Luna, D. Dragoe, K. Wang, P. Beaunier, E. Kowalska, B. Ohtani, D. Bahena Uribe, M. A. Valenzuela, H. Remita and C. Colbeau-Justin, J. Phys. Chem. C, 2017, 121, 14302-14311.

39 T. A. Kandiel, A. A. Ismail and D. W. Bahnemann, Phys. Chem. Chem. Phys., 2011, 13, 20155-20161.

40 J. Serafin, L. Soler, D. Vega, Á. Rodríguez and J. Llorca, Chem. Eng. J., 2020, 393, 124701.

41 F. Della Foglia, G. L. Chiarello, M. V. Dozzi, P. Piseri, L. G. Bettini, S. Vinati, C. Ducati, P. Milani and E. Selli, Int. J. Hydrogen Energy, 2014, 39, 13098-13104.

42 G. L. Chiarello, L. Forni and E. Selli, Catal. Today, 2009, 144, 69-74.

43 R. Su, M. M. Forde, Q. He, Y. Shen, X. Wang, N. Dimitratos, S. Wendt, Y. Huang, B. B. Iversen, C. J. Kiely, F. Besenbacher and G. J. Hutchings, J. Chem. Soc., Dalton Trans., 2014, 43, 14976-14982.

44 J. Polleux, N. Pinna, M. Antonietii and M. Niederberger, $A d v$. Mater., 2004, 16, 436-439. 